\title{
Pulmonary aspergillosis in a patient with COPD due to PEG-interferon and ribavirin therapy for chronic hepatitis C
}

\author{
Ersoy Ozdal*, Vardareli Eser, Getiren Seyhmus, Tozun Nurdan \\ Internal Medicine-Gastroenterology Department, Acibadem University, Istanbul, Turkey; \\ *Corresponding Author: ozdal.ersoy@acibadem.edu.tr, ozdalersoy@gmail.com
}

Received 25 January 2013; revised 26 February 2013; accepted 12 March 2013

\begin{abstract}
This is a case of $\mathbf{5 2}$ years old male patient with chronic obstructive pulmonary disease (COPD) who developed semi-invasive pulmonary aspergillosis while on pegylated-interferon alpha-2a and for chronic hepatitis $C$ infection. This complication is very rarely seen during interferon therapy for other any purposes. Pulmonary aspergillosis in particular group of chronic hepatitis C patients under immunosupressive therapy should also be in mind and interdisciplinary medical cooperation might be needed during the treatment for immune-suppressed patients. Pulmonary aspergillosis is also discussed from many aspects in the light of the literature.
\end{abstract}

Keywords: Hepatitis C; Aspergillosis; Interferon

\section{INTRODUCTION}

This is a case of 52 years old male patient with chronic obstructive pulmonary disease (COPD) who developed semi-invasive pulmonary aspergillosis while on pegylated-interferon alpha-2a and ribavirin for chronic hepatitis $\mathrm{C}$ infection. He was hospitalised and recovered by discontinuation of all anti-viral therapy and getting caspofungin bid intravenously for 3 weeks. Myelosuppresant effect of interferon and ribavirin permits this very rarely seen opportunistic infection in this immune-supressed patient and we also postulate that concomitant presence of COPD and use of low dose sterod inhalers has become another additive risk factors for this opportunistic infection. Although a new case of combined fungal infection (pulmonary aspergillosis and esophageal candidiasis) in a patient with decompansated cirrhosis has been recently published, up to the literature review, this is the first case of pulmonary aspergilosis in a hepatitis $\mathrm{C}$ patient treated with pegylated interferon (PEG-IFN) and ribavirin (RBV). Because many rare but serious complications can occur during the treatment of $\mathrm{HCV}$ with Peg - IFN + RBV treatment, patients and their clinicians should be in close contact especially when unusal symptoms occur or if the patient has other comorbid or concurrent diseases in order to prevent fatal ends. Aim of this article is to report this very rarely seen case and to increase the awareness of pulmonary aspergillosis in particular group of chronic hepatitis $\mathrm{C}$ patients under immunosupressive therapy and stressing the value of interdisciplinary medical cooperation and treatment for immune-suppressed patients by reviewing the pulmonary aspergillosis from many aspects in the light of the literature.

\section{CASE}

Chronic hepatitis $\mathrm{C}$ infection with normal transaminase levels (anti-HCV: positive, HCV-RNA: 685,447 IU/ $\mathrm{mL}-1,850,707$ copy $/ \mathrm{mL}$, genotype $1 \mathrm{~b}$ ) was diagnosed in an asymptomatic 52 years old man, (living in a rural part of Turkey) during his control visit of benign prostate hypertrophy in May 2010. He had a previous history of chronic obstructive pulmonary disease (COPD) with bronchiectasis, for which he used fluticasone plus salmaterol inhalation with oral/inhaler prednisolone treatment. Patient was nondiabetic and normotensive. He had drunk alcohol moderately and had no other addiction. He was consultated with his pulmonologist first, for any kind of contrindications to immunosuppressive treatment then he was allowed to take Peginterferon $\alpha$-2a (PEG-IFN) 180 mcg +Ribavirin $1000 \mathrm{mg}$ /day combination therapy for chronic hepatitis C on September 2010. On pyhsical examination, there were mild signs of bronchoconstriction (coarse pulmonary rales and prolonged expirium) without any signs of exacerbation (fever, malaise, productive cough, short of breath). Complete blood count, sedimentation rate and CRP, standard biochemistry and electrolytes, protein electrophoresis, thyroid functions, 
anti-smooth muscle antibody, anti-LKM1 antibody, lactate dehydrogenase, serum iron, ferritin and total iron binding capacity were all within normal ranges. Weekly for 4 weeks then monthly controlled complete blood count and liver function tests in addition with 1 st and 3rd month HCV RNA levels were evaluated during routine follow-up visits. Patient suffered from fatigue along with weight loss and loss of appetite starting from the first week of treatment, gradually increasing in intensity. During follow up blood counts and liver function tests were in normal range and HCV RNA were found to be negative on 1st and 3rd month of treatment. During the 4 th month of the treatment, he was prescribed moxifloxacin treatment for 10 days due to a respiratory tract infection from his local hospital. At the 18th week of therapy the patient complained about progressive fatigue, nausea and being unable to walk along with weight loss of more than $10 \mathrm{~kg}$ in one month. Finally he was referred to our clinic from his local hospital, in a lethargic appearance with intensive productive cough. As he was evaluated in our out-patient clinic, he was immediately hospitalised and RBV anf PEG-IFN therapy was cessated with no doubt. Physical examination revealed pallor, fine tremors of hands, dry skin and, decreased breath sounds, coarse rales along with fine rales and widespread ronchi were perceived on lung examination. On cardiac examination there was only tachycardia. Widespread oral thrush was seen. Blood examination revealed that CRP: 11.8 mg/dL, ESR: 83/min, Hemoglobin: $10.6 \mathrm{mg} / \mathrm{dL}$, Hematocrit: $33.5 \%$, Leukocyte: $8700 / \mathrm{mL}$ (differential count is normal), Albumin: $3.0 \mathrm{~g} / \mathrm{dL}$, and liver enzymes and electrolytes were in normal range. Fever was $38.3^{\circ} \mathrm{C}$. There was no cyanosis. Intense bronchiectatic appearance and pneumonic infiltrations were noticed in the chest X-ray. Fluconazole $400 \mathrm{mg}$ /day, nystatin oral suspension four times daily and ceftriaxone $1 \mathrm{~g}$ /day bid were started under the pre-diagnosis of acute pulmonary infection. Quantiferon level, aerobic-anaerobic-fungal blood and sputum cultures, and acid-resistant bacillus investigation in sputum were searched. High-resolution computed-tomography of thorax revealed that there were an increase in diameter of trachea and bilateral main bronchi; bilateral widespread cystic and tubular bronchiectasis; thickening of walls of bronchi and frequent "tree-in-bud" appearances in both lungs; left lung located densities, which the biggest one is $39 \times 31 \times 45 \mathrm{~mm}$ size, in which air-fluid levels and air trappings exist (Figure 1); bilateral some air cysts and mosaic perfusion pattern; a $60 \times 29 \times 58 \mathrm{~cm}$ sized, large based, pleural located calcified mass. Findings were evaluated as chronic obstructive lung diseaseemphysema. In his detailed history, he also stated the presence of fungal molds on the walls of his bedroom for the last $1-2$ months because of the problems on the water drainage of the roof. Along with this history and the

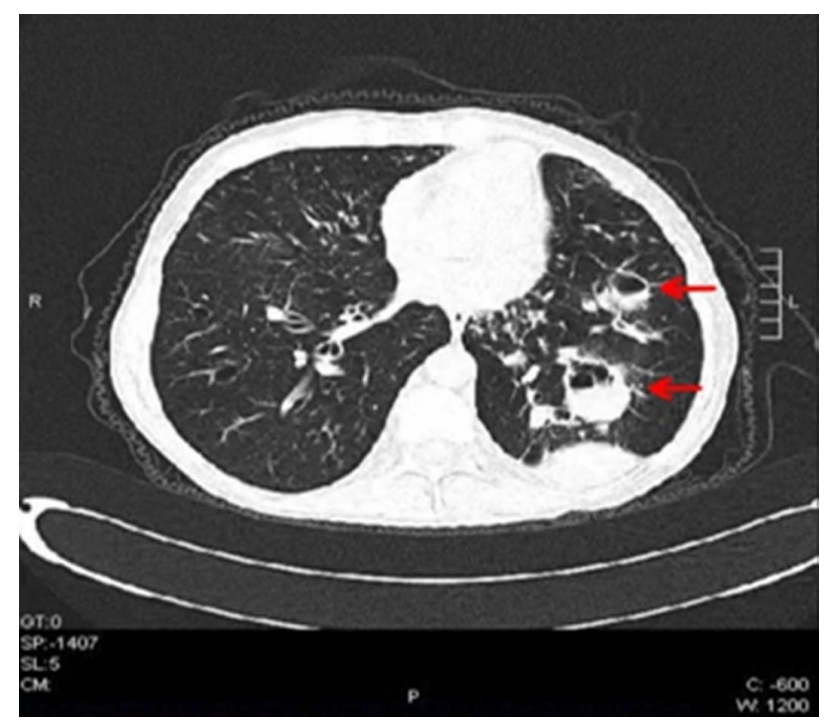

Figure 1. Left lung located densities with air-fluid levels (arrow-heads).

cystic and tubular bronchiectasis, densities which contain air trappings and air-fluid levels and being able to continue with bronchi were assessed as invasive bronchopulmonary aspergillosis or tuberculosis. The pleural mass lesion had existed in his previous CT scans and assessed as benign. Widespread "tree-in-bud" appearance seen in both lungs was considered as mucous plugs developed secondarily due to bronchiectasis. Surrounding these appearances there were focal ground-glass regions and were evaluated as bronchiolitis-bronchopneumonia. After these findings, serum tests for Aspergillus galactomannan antigens were searched and antibiotics and antifungals were changed to caspofungin $50 \mathrm{mg}$ /day and piperacillin plus tazobactam $4.5 \mathrm{~g}$ tid. Beta receptor agonists and oxygen support were started for his dyspnea and hypoxemia. In addition, TSH and cortisol levels were investigated. Patient was found to have subclinical hypothyroidism. Thyroid hormone and a short-term folic acid replacement therapy was started. There were not any acid-resistant bacteria's in sequential sputum samples. Quantiferon test, M. tuberculosis PCR and aspergillus PCR tests were found to be negative. The result of all blood cultures were negative for any pathogen. Result of Aspergillus galactomannan antigen level was $0.67 \mathrm{pg} / \mathrm{dL}$ (normal level: $<0.50 \mathrm{pg} / \mathrm{dL})$. Oral thrushes gradually diminished and cough, expectoration and fever all disappeared at the second week of treatment. Aspergillus galactomannan antigen level was decreased to $0.13 \mathrm{pg} / \mathrm{dL}$ at the end of the 3rd week of the treatment. The patient was discharged after 3 weeks of treatment when his general status and respiratory symptoms were improved. PEG-IFN/RBV treatment never started again. His subsequent control visit 2 weeks after discharge showed normal blood tests and improved radiological appearance (Figure 2) and ge- 
neral status without any pulmonary symptoms. He is still on close follow-up and HCV-RNA level is still being negative for 18 months after the cessation of the anti-viral treatment.

\section{DISCUSSION}

Patients with advanced or acute liver disease have an increased susceptibility to fungal infections for the reason of the disease itself and in relation to a significant impairment of neutrophil functions, increased intestinal permeability, frequent need to use of corticosteroids and broad-spectrum antibiotics for other basic diseases, malnutrition, increasing performance of invasive procedures and the degraded immune function by the immunemodulating and supressing drugs [1,2]. Fiuza et al. described significant defects in neutrophil migration and phagocytosis with advenced cirrhosis [3]. Aspergillosis is one of these fungal infections and can be a major cause of morbidity and mortality in these immunocompetent patients with liver diseases especially following liver transplantations [4,5]. Very recently, Probrucin et al. published a case of combined fungal infection in a patient with decompansated liver disease due to chronic hepatitis $\mathrm{C}$ and alcohol [6]. However, fungal infections have no characteristic clinical findings and have low positivite ratio for culture; it is easy to misdiagnose and result in delayed treatment and even in lethal ends. Combination of long-acting pegylated interferferon alpha (PEG-IFN $\alpha$ ) and ribavirin (RBV) is the current and the best recommended treatment option to treat chronic hepatitis $\mathrm{C}$ [7]. However, attention should be always given to the possibility of bacterial and fungal infections during treatment with these drugs for hepatitis $\mathrm{C}$ infection. Hepatitis $\mathrm{C}$ alone suppress both humoral and cell-mediated immunity

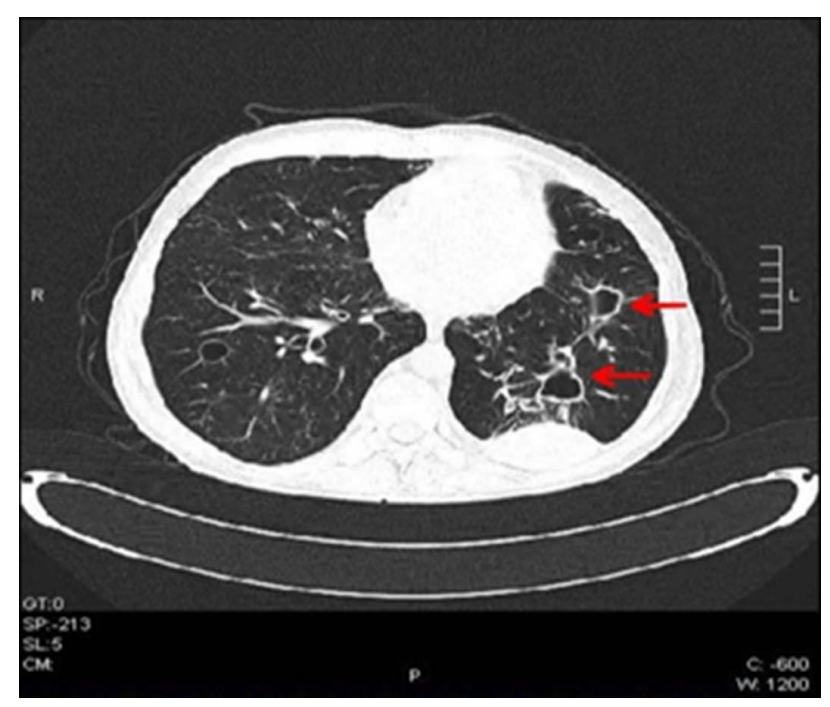

Figure 2. Regressed lung densities after 2 weeks of anti-fungal treatment. of the patient but these drugs also cause additive myelodepressant and immune modulating effects leading to increased risk of infections. Older age and poorly controlled diabees mellitus are also associated with an increased risk of infections during $\mathrm{HCV}$ treatment [8]. Besides having Hepatitis $\mathrm{C}$ infection and started PEG-IFN/RBV treatment, our patient was carrying other important risk factors for increased possibilityof infections during this treatment. He had been treated for pulmonary tuberculosis 20 years ago and had some chronic parenchymal sequlea in his lungs and he was currently taking drugs [inhaler bronchodilator s and corticosteroids] for COPD for the last 20 years and had many hospitalisations due to COPD exacerbations. He was also living in another city far from where the treatment will be started and followups are planned to take place [ 1 hour by plane, $7-8$ hours by car], due to less humidiy where he feels himself more comfortable in terms of respiratory problems. Therefore reactivation of tuberculosis or worsening COPD and the problems of the distance in between the patient and his hepatologist (by means of control periods and emergency situations) were all noted to be possible important difficulties and complications to be faced with during PEG-IFN and RBV treatment. However, finally he was started to take anti-viral therapy after his pulmonologist has convinced that he had no more active tuberculosis nor uncontrolled COPD but advised closer contacts and frequent follow-ups throughout the treatment period. Our patient has been taking the anti-viral therapy for the last 4 months when his symptoms; cough, fever, general status deterioration had first appeared. He first went to local hospital however, despite the presence of all these mentioned predisposing factors, patient was not considered by the physician as a patient at risk for important fungal infection because of negative HCV- RNA level and normal hemoglobin and neutrophil levels at the end of 3rd month control and considered as bacterial pulmonary infection and was prescribed an empiric antibiotic without cessating PEG-IFN/RBV and performing further evaluations, and this leaded to a lack and delay in diagnosis of pulmonary aspergilosis. Generally the progression of pulmonary aspergillosis develops quickly, thus our case got worse under the given antibiotherapy because of the misdiagnosis of bacterial pnemonia.

Aspergillus species are ubiquitous molds found on dead or decaying organic matter. Aspergillus may cause a broad spectrum of disease in the human host, ranging from hypersensitivity reactions to direct angioinvasion and the transmission of fungal spores to the human host is via airborne route. Aspergillus primarily affects the lungs. The pulmonary aspergillosis manifestations are determined by the patient's immune status and the presence of underlying disease [9]. It causes 4 main syndromes, including allergic bronchopulmonary aspergillo- 
sis, chronic necrotizing Aspergillus pneumonia (or chronic necrotizing pulmonary aspergillosis or semiinvasive pulmonary aspergilosis), airway-invasive aspergilosis, aspergilloma, and angioinvasive aspergillosis with systemic involvement. The pulmonary aspergilosis is a severe disease especially seen among immunosupressed patients and in patients with well established risk factors such as, hematological malignancies, organ transplantation, or HIV. Semi-invasive aspergillosis, which is the final diagnosis of our case, is a rare locally destructive form of aspergillosis. Diabetes mellitus, advanced age, prolonged corticosteroid therapy, COPD, a history of pulmonary tuberculosis, previous pulmonary resections and immunsuppression are knownn risk factors for the development of this form of pulmonary aspergilosis [10]. Mostly occur under neutropenia but also occur in non-neutropenic patients, e.g., those with steroid-associated immunosupression. Although seen as a common side effect and a cause of dose reductions of PEG-IFN therapy, neutropenia was not observed in our patient. Recently, Roomer et al., showed that infections during treatment with PEG-IFN/ RBV are not associated with neutropenia and they, also stated the older patients and patients with uncontrolled diabetes had a greater risk of developing infections during HCV treatment [8]. Our 52 years old case with noneutropenic but having concomitant COPD, had also mentioned about taking notice of the mould on the walls of his bedroom due to recently occured roof water-proofing problem. Because of all these predisposing factors, as soon as our case had been referred to us by his local physician to improve his prognosis, we immediately postulate that our patient might have acquired pulmonary fungal infection.

As mentioned before, the frequency of pulmonary aspergilosis increases in patients with COPD. Oral or inhaler steroid use in conjunction with recurrent courses of systemic antibiotics also increases the risk. Most COPD patients are used to take antimicrobial drugs as soon as clinical manifestations of the respiratory tract infections start. Pulmonary aspergilosis is commonly presented as a respiratory tract infection, but with poor response to antimicrobial treatment and the delay in its diagnosis is one of the main causes of its high lethality [11]. Our case was only using inhaler bronchodilators when hepatitis $\mathrm{C}$ treatment was started. When sypmtoms of the respiratory tract infection occurred, he was given levofloxacin but he did not get any response to the treatment in 10 days and even got worse, then he was referred to our clinic and was eventually hospitalised. Clinical symptoms of pulmonary aspergilosis are cough with expectoration, bloody sputum, fever, dyspnea and weight loss and loss of appetite which all show similarity with the symptoms of exacerbations of COPD. All these non-specific symptoms were also observed in our patient at the beginning, however, the ini- tiation of additional diagnostic examinations was delayed in the local hospital because of a low clinical suspicion of the physician. However, not only our case is having chronic viral hepatitis $\mathrm{C}$ and a history of tuberculosis, pulmonary resections and COPD, but also sometimes needs to use inhaler or oral corticosteroid and has just started PEG-IFN/RBV immunomodulating anti viral therapy, so it is very important for the physicians to be familiar with complicating fungal infections. The delay in the diagnosis of pulmonary aspergillosis is one of the main causes of the severe debilitating state in our case. Thus, once suspected, respiratory secretion cultures and imaging modalities of the lung should be quickly obtained and galactomannan test should be determined. After cessating Peg-IFN and RBV therapy, all these tests were obtained from our patient as soon as he admitted to the hospital. However, the diagnosis of pulmonary aspergilosis is often difficult. Although exact diagnosis needs the histological evidence of invasion of lung tissue by septal hyphae, consisting with aspergillus, it is often difficult to obtain these evidences and isolate the fungus from the sputum or the broncoalveolar lavage examinations and lung parenhymal tissue [1]. Therefore the diagnostic process should be continued with thoracic imaging and microbiological fungus antigen quantifications.

Thoracic CT-scan features of pulmonary aspergilosis generally include multiple nodular opacities and areas of consolidation with or without cavitation or adjacent pleural thickening [12]. These are all similar radiological features seen in our case. Radiological (high resolution computerised tomography-HRCT) findings in our case consisted of an increase in diameter of trachea and bilateral main bronchi; bilateral widespread cystic and tubular bronchiectasis; thickening of walls of bronchi associated with a "tree-in-bud" appearances in both lungs; left lung located densities, which the biggest one is $39 \times 31 \times 45$ $\mathrm{mm}$ size, in which air-fluid levels and air trappings exist; bilateral some air cysts and mosaic perfusion pattern; a $60 \times 29 \times 58 \mathrm{~cm}$ sized, large based, pleural located calcified mass. However, the pleural mass lesion had existed in his previous CT scans and assessed as benign.

The detection of galactomannan (GM) is a new valuable test for the diagnosis of invasive aspergilosis. GM is a polysaccharide fungal cell wall component that is released during tissue invasion by aspergillus hyphae and can be detected in body fluids [1]. GM detection is frequently seen in sputum and bronchoalveolar lavage (BAL) fluid and rarely in blood. Our experience with this case confirmed the GM detection only in blood which seems contrary to the literature. Therefore this test (GM detection in serum) could be considered as an alternative option in patients whom no exact diagnosis could be performed by sputum or other body fluids. Repeated levels of GM antigens could also be used in order to follow-up 
the patient.

Empiric anti-fungal therapy should be rapidly carried out while the etiology evidence is actively searched especially in patients with high risk of invasive fungal infections. In our case, anti-fungal treatment has started rapidly as soon as the suspicion occurred after radiological findings while the GM, antigens and cultures were being carried out. Early therapy is important in order to have favorable outcomes. Outcome is better if patients have well-controlled underlying disease and granulocyte count greater than 1000 [13] Amphotericin B, fluconazole, itraconazole and variconazole have been used with success in several cases of pulmonary or invasive aspergillosis [2] with different effective rates. In 2006, Glasmacher et al. reported the efficacy and safety of Caspofungin $(\mathrm{CF})$ in a large unselected population of patients with fungal infections in Germany [14]. CF, which is the first-in-echinocandin class, is an antifungal drug with a broad spectrum of activity and excellent tolerbility. We also administered CF to our case as the choice of treatment because of its availability and less toxicity and continued for 3 weeks without any reportable side effects. Of importance CF has particularly favourable pharmacokinetic properties since they are not metabolized by cytochrome P450 enzyme system [1].

\section{CONCLUSION}

As a summary this case highlights first that; pulmonary aspergillosis can occur in patients with chronic hepatitis $\mathrm{C}$ infection during the immunomodulating treatment period and the riskof this fungal infection increases with concomitant presence of COPD and use of the steroids. Physicians should be aware in recognizing this infection in this particular group of patient and antiviral therapy should be prescribed with great caution. And second, it is valuable to recognize that, in immunocompromised patients with COPD, aspergillus infection should be considered as an important differential diagnosis in respiratory infections that are difficult to treat with empiric antibiotherapy [15]. Unfortunately, there are no trials documenting that aspergillus infection can be prevented in risk groups [16], but its early diagnosis and promptly given treatment together with reduction in risk factors, appears significantly improve the prognosis of the patient.

\section{REFERENCES}

[1] Falcone, M., Massetti, A.P., Russo, A., Vullo, V. and Venditti, M. (2011) Invasive aspergillosis in patients with liver disease. Medical Mycology, 49, 406-413. doi:10.3109/13693786.2010.535030

[2] Hou, Z., Tan, D., Liu, G., Xie, Y., Li, C., Xie, J., et al. (2010) Clinical characteristics and therapeutic analysis of invasive fungal infection in chronic severe hepatitis patients. Journal of Central South University. Medical Sciences, 35, 537-542.

[3] Fiuza, C., Salcedo, M., Clemente, G. and Tellado, J.M. (2000) In vivo neutrophil dysfunction in cirrhotic patients with advanced liver disease. The Journal of Infectious Diseases, 182, 526-533. doi:10.1086/315742

[4] Tsiodras, S., Zafiropoulou, R., Giotakis, J., Imbrios, G., Antoniades, A. and Manesis, E.K. (2004) Deep sinus aspergillosis in a liver transplant recipient successfully treated with a combination of caspofungin and voriconazole. Transplant Infectious Disease, 6, 37-40. doi:10.1111/j.1399-3062.2004.00039.x

[5] Paya, C.V. (2001) Prevention of fungal and hepatitis virus infections in liver transplantation. Clinical Infectious Diseases, 33, S47-S52. doi:10.1086/320904

[6] Hoofnagle, J.H. and Seeff, L.B. (2006) Peginterferon and ribavirin for chronic hepatitis C. The New England Journal of Medicine, 355, 2444-2451. doi:10.1056/NEJMct061675

[7] Porobcin, S., Porubcinova, I., Viraq, L., Stammova, E., Vyhankova, V. and Paralicova, Z. (2012) Invasive pulmonary aspergillosis and esophageal candidiasis in a patient with decompansated liver cirrhosis due to chronic hepatitis $\mathrm{C}$ and alcohol. Klinicka Microbiologie a Infeckni Lekarstvi, 18, 17-21.

[8] Roomer, R., Hansen, B.E., Janssen, H.L. and de Knegt, R.J. (2010) Risk factors for infection during treatment with peginterferon alfa and ribavirin for chronic hepatitis C. Hepatology, 52, 1225-1231. doi:10.1002/hep.23842

[9] Franquet, T., Müller, N.L., Giménez, A., Guembe, P., de La Torre, J. and Bagué, S. (2001) Spectrum of pulmonary aspergillosis: Histologic, clinical, and radiologic findings. Radiographics, 21, 825-837.

[10] Cabral, F.C., Marchiori, E., Zanetti, G., Takayassu, T.C. and Mano, C.M. (2009) Semi-invasive pulmonary aspergillosis in an immunosuppressed patient: A case report. Cases Journal, 12, 40. doi:10.1186/1757-1626-2-40

[11] Valle, J.M., González-Barcala, F.J., Alvarez-Dobaño, J.M. and Valdés, L. (2010) Invasive pulmonary aspergillosis in chronic obstructive pulmonary disease. Revista Médica de Chile, 138, 612-620.

[12] Aquino, S.L., Kee, S.T., Warnock, M.L. and Gamsu, G. (1994) Pulmonary aspergillosis: Imaging findings with pathologic correlation. American Journal of Roentgenology, 163, 811-815. doi:10.2214/ajr.163.4.8092014

[13] Patterson, T.F., Miniter, P., Ryan, J.L. and Andriole, V.T. (1988) Effect of immunosuppression and amphotericin B on Aspergillus antigenemia in an experimental model. The Journal of Infectious Diseases, 158, 415-422. doi:10.1093/infdis/158.2.415

[14] Glasmacher, A., Cornely, O.A., Orlopp, K., Reuter, S., Blaschke, S., Eichel, M., et al. (2006) Caspofungin treatment in severely ill, immunocompromised patients: A case-documentation study of 118 patients. Journal of Antimicrobial Chemotherapy, 57, 127-134. doi:10.1093/jac/dki410

[15] Ali, Z.A., Ali, A.A., Tempest, M.E. and Wiselka, M.J. 
(2003) Invasive pulmonary aspergillosis complicating chronic obstructive pulmonary disease in an immunocompetent patient. Postgraduate Medical Journal, 49, 7880. doi:10.4103/0022-3859.922
[16] Kriengkauykiat, J., Ito, J.I. and Dadwal, S.S. (2011) Epidemiology and treatment approaches in management of invasive fungal infections. Journal of Clinical Epidemiology, 3, 175-191. doi:10.2147/CLEP.S12502 